\title{
Transferrin-Liposome-Mediated Systemic p53 Gene Therapy in Combination with Radiation Results in Regression of Human Head and Neck Cancer Xenografts
}

\author{
LIANG XU, KATHLEEN F. PIROLLO, WEN-HUA TANG, ANTONINA RAIT, and ESTHER H. CHANG
}

\begin{abstract}
The use of cationic liposomes as nonviral vehicles for the delivery of therapeutic molecules is becoming increasingly prevalent in the field of gene therapy. We have previously demonstrated that the use of the transferrin ligand (Tf) to target a cationic liposome delivery system resulted in a significant increase in the transfection efficiency of the complex [Xu, L., Pirollo, K.F., and Chang, E.H. (1997). Hum. Gene Ther. 8, 467-475]. Delivery of wild-type (wt) p53 to a radiation-resistant squamous cell carcinoma of the head and neck (SCCHN) cell line via this ligand-targeted, liposome complex was also able to revert the radiation resistant phenotype of these cells in vitro. Here we optimized the Tf/liposome/DNA ratio of the complex (LipT) for maximum tumor cell targeting, even in the presence of serum. The efficient reestablishment of wtp53 function in these SCCHN tumor cells in vitro, via the LipT complex, restored the apoptotic pathway, resulting in a significant increase in radiation-induced apoptosis that was directly proportional to the level of exogenous wtp53 in the tumor cells. More significantly, intravenous administration of LipT-p53 markedly sensitized established SCCHN nude mouse xenograft tumors to radiotherapy. The combination of systemic LipT-p53 gene therapy and radiation resulted in complete tumor regression and inhibition of their recurrence even 6 months after the end of all treatment. These results indicate that this tumor-specific, ligand-liposome delivery system for p53 gene therapy, when used in concert with conventional radiotherapy, can provide a new and more effective means of cancer treatment.
\end{abstract}

\section{OVERVIEW SUMMARY}

A transferrin-cationic liposome system was optimized for efficient and tumor-specific gene delivery both in vitro and in vivo. The replacement of wild-type $\mathrm{p53}$ by lipT-p53 restored the wtp53 function and apoptotic pathway. The systemic delivery of the LipT-p53 to established nude mouse xenograft model of human squamous cell carcinoma of the head and neck markedly sensitized the tumor to radiation therapy. The combination of system ic p53 gene therapy and conventional radiotherapy resulted in tumor regression and inhibition of their recurrence long-term. This strategy may provide a more effective treatment modality for human head and neck cancer.

\section{INTRODUCTION}

$\mathbf{W}$ ILD-TYPE (wt) p53 plays a crucial role in apoptotic pathways leading to cell death (reviewed in Bates and Vousden, 1999). The lack of functional p53 is believed to contribute to the failure of many cancers to respond to conventional therapies (reviewed by Ruley, 1996; Roth, 1998). For example, the presence of mutant (mt) p53 has been shown to correlate with increased radiation resistance in some human tumors and cell lines, possibly because of the loss of the p53-dependent apoptotic pathway (Lee and Bernstein, 1993; O'Connor et al., 1993; McIlwrath et al., 1994; Bristow et al., 1996; Nielson and Maneval, 1998; Meng and El-Deiry, 1999). Therefore, reestablishing wt p53 function in tumor cells may restore the p53-me-

Department of Otolaryngology, Head and Neck Surgery, Lombardi Cancer Center, Georgetown University Medical Center, Washington, D.C. 20007. 
diated apoptotic pathway, resulting in more efficient treatment modalities.

Progress has been made toward developing nonviral, pharmaceutical formulations of genes for in vivo human therapy, particularly cationic liposome-mediated gene transfer systems (Felgner et al., 1995; Templeton et al., 1997; Huang and Viroonchatapan, 1999). However, liposomal systems in general suffer from low transfection efficiencies when compared with those observed with viral vector delivery systems. The transfection efficiency of cationic liposomes can be dramatically increased when a ligand recognizing a cell surface receptor is included in the complex. The presence of the ligand facilitates the entry of the DNA-liposome complex into the cell through the initial binding of ligand by its receptor on the cell surface, followed by internalization of the bound complex via receptormediated endocytosis (Cheng, 1996), a highly efficient internalization pathway present in eukaryotic cells (Christiano and Curiel, 1996; Wagner, 1999). We have previously reported that the addition of the transferrin (Tf) ligand to a cationic liposome complex resulted in an in vitro transfection efficiency of $70-80 \%$ in squamous cell carcinoma of the head and neck (SCCHN) as compared with only 5-20\% transfection by the cationic liposome complex without Tf (L. Xu et al., 1997). Transferrin receptor (TfR) levels are known to be elevated in various types of cancer cells including breast (Inoue et al., 1993) and prostate cancers (Keer et al., 1990), even those prostate cancer cell lines derived from human lymph node and bone metastases (Grayhack et al., 1979; Chackal-Roy et al., 1989; Rossi and Zetter, 1992), as well as squamous cell carcinomas (Thorstensen and Romsio, 1993; Miyamoto et al., 1994), and correlate with the aggressive or proliferative ability of tumor cells (Elliott et al., 1993).

Squamous cell carcinoma of the head and neck is one of the most difficult forms of cancer to treat successfully. Approximately 40,000 new cases are diagnosed in the United States each year, with more than 12,000 individuals succumbing to this devastating disease annually (Landis et al., 1998). Despite advances in the preferred forms of therapy, i.e., surgery and radiation, and now even chemotherapy, no major improvement in efficacy and survival has occurred over the past several decades. Approximately $70 \%$ of patients with advanced disease (stage III-IV) will still die from their illness, primarily from recurrent disease, which occurs in more than $60 \%$ of patients (Dimery and Hong, 1993). Even $25 \%$ of those with early stage disease (stage I-II) are incurable (van Dongen and Snow, 1997). Resistance to ionizing radiation by a subset of the tumor cells is believed to account for a significant portion of these clinical failures. Therefore, a new treatment modality that results in sensitization of these tumors to standard radiation therapy would have a marked effect on the clinical outcome of this disease for many patients.

In our previous report (L. Xu et al., 1997), we demonstrated that wt p53 was efficiently delivered to SCCHN tumor cells in vitro by the $\mathrm{Tf}$-liposome complex. The high-level expression of this exogenous wt p53 in the Tf-liposome-DNA-transfected cells resulted in the reversion of the radioresistant phenotype of the SCCHN cells in a DNA dose-dependent manner. In the present study, we further optimized the transferrin-cationic liposome system for systemic p53 gene therapy of SCCHN and demonstrate that the transduced wt p53 restored wt p53 func- tion in these cells, leading to increased radiation-induced apoptosis in a 553 DNA-dependent manner. These findings indicate that the reversal of the radiation resistance of the SCCHN cells observed in vitro in our previous report was due to this restoration of the p53-dependent apoptotic pathway.

Most significantly, we examined the ability of transfected wt p53 to sensitize SCCHN to radiation in vivo. Our findings demonstrate that this transferrin-cationic liposome system could preferentially deliver the p53 gene to preestablished SCCHN xenograft tumors and sensitize them to radiotherapy, resulting in long-term tumor regression in animals receiving the combination therapy. This sensitization of SCCHN tumors, through the combination of systemic p53 gene therapy and ionizing irradiation, has the potential for use as a new therapeutic modality for the treatment not only of initial malignancies, but also for the larger challenge of recurrent disease.

\section{MATERIALS AND METHODS}

\section{Cell culture}

Human head and neck cell line JSQ-3, derived from a tumor of the nasal vestibule that failed radiotherapy, was a generous gift from R. Weichselbaum (University of Chicago; Weichselbaum et al., 1993). The cell line was maintained in minimum essential medium with Earle's salts (EMEM) with supplem ents as previously described (L. Xu et al., 1997).

\section{Plasmids}

The Escherichia coli lacZ gene, driven by a simian virus 40 (SV40) promoter in expression plasmid pSVb, and the firefly luciferase gene driven by the cytomegalovirus (CMV) promoter in plasmid pCMVLuc, were employed as reporter genes (both from Promega, Madison, WI). The p53 expression plasmid pCMVp53 contains the 1.7-kb human wt p53 cDNA under the control of the CMV promoter, followed by the SV40 polyadenylation signal. The control plasmid pCMVpRo contains the same p53 cDNA in the reversed orientation, also under the control of the CMV promoter. All the plasmids were expanded in $E$. coli DH5 $\alpha$ and purified with plasmid Mega/Giga kits (Qiagen, Valencia, CA). The purified plasmids were quantified spectrophotometrical ly with $A_{260} / A_{280}$ values of approxim ately 1.95 . Agarose gel $(0.8 \%)$ electrophoresis confirmed that $>95 \%$ of the plasmid DNA was supercoiled.

\section{Preparation of transferrin-liposome-DNA complex}

Cationic liposomes consisting of 1,2-dioleoyl-3-trimethylammoniumpropane (DOTAP) and dioleoylphosphatidylethanolamine (DOPE) (Avanti Polar Lipids, Alabaster, AL) were prepared as previously described (L. Xu et al., 1997). The final concentration of liposomes was $2 \mu \mathrm{mol} / \mathrm{ml}$. Holo-transferrin (Tf, iron saturated; from Sigma, St. Louis, MO) was dissolved in pure water at $5 \mathrm{mg} / \mathrm{ml}$. The $\mathrm{Tf}$-liposome-DNA complex for in vitro experiments was prepared as described except that the serumfree medium EMEM was used instead of Hanks' balanced salt solution (HBSS) (L. Xu et al., 1997). The prepared Tf-liposome (designated LipT)-DNA complex was used for in vitro cell transfection freshly within $1 \mathrm{hr}$ of preparation, although on 
the basis of transfection efficiencies it was found to be stable for at least $24 \mathrm{hr}$. Agarose gel electrophoresis was employed to assess the DNA retardation by complexing with LipT.

\section{Optimization of LipT system by luciferase assay}

The LipT system was optimized for JSQ-3 transfection using the luciferase assay. JSQ- 3 cells $\left(5 \times 10^{4} /\right.$ well $)$ were plated in a 24-well tissue culture plate. Twenty-four hours later, the cells were washed once with EMEM without serum, and 0.3 $\mathrm{ml}$ of EMEM without serum or antibiotics was added to each well. EMEM $(0.2 \mathrm{ml})$ with the Tf-liposome-pCMVLuc (LipT-Luc) complex containing $1.0 \mu \mathrm{g}$ of plasmid DNA complexed with different amounts of cationic liposome and transferrin was added to the cells, usually in triplicate. After a 5-hr incubation at $37^{\circ} \mathrm{C}$ and $5 \% \mathrm{CO}_{2}, 0.5 \mathrm{ml}$ of EMEM, supplemented with $20 \%$ fetal bovine serum and hydrocortisone (1 $\mu \mathrm{g} / \mathrm{ml})$, was added to each well. Twenty-four hours later, the cells were washed once with phosphate-buffered saline (PBS), lysed with $1 \times$ reporter lysis buffer (100 $\mu \mathrm{l} /$ well; Promega), and the expressed luciferase activities were measured with the luciferase assay system (Promega) on a luminometer (Xu et al., 1999). A recombinant firefly luciferase (Promega) standard was used at each measurement so as to convert the luminometer readings of relative light units (RLU) to the amount of luciferase equivalent expressed. Protein concentrations of the cell lysates were measured with a DC protein assay kit (Bio-Rad, Hercules, CA). The results were expressed as micrograms of luciferase equivalent per milligram of total protein.

\section{In vitro transfection of JSQ-3 cells by LipT-pSVb}

A quantitative $\beta$-galactosidase colorimetric assay was employed as described previously (L. Xu et al., 1997). Purified $\beta$ galactosidase (Boehringer Mannheim, Indianapolis, IN) was used as standard. The results were expressed as milliunits (mU) of $\beta$-galactosidase equivalent per milligram of total protein. For histochemical studies of Tf-liposome-pSVb transfection, $60 \%$ confluent JSQ-3 cells in a 24-well tissue culture plate were transfected for $5 \mathrm{hr}$ with $1.2 \mu \mathrm{g}$ of $\mathrm{pSVb}$ with or without LipT as described above. After an additional 2 days in culture, the cells were fixed and stained with 5-bromo-4-chlor o-3-indolyl$\beta$-D-galactopyranoside (X-Gal), and transfection efficiency was calculated as the percentage of blue-stained cells as previously described (L. Xu et al., 1997, 1999).

\section{Western blot analysis}

To determine the DNA-dose response of p53 gene expression, $2 \times 10^{5}$ JSQ- 3 cells/well were plated in a six-well tissue culture plate. After $24 \mathrm{hr}$, cells were washed once with EMEM without serum and antibiotics, transfected with $1 \mathrm{ml}$ of transfection solution containing LipT-p53 or LipT-pRo containing up to $4 \mu \mathrm{g}$ of plasmid DNA per $2 \times 10^{5}$ cells, at the ratio of 1 $\mu \mathrm{g}$ of DNA per $10 \mathrm{nmol}$ of liposome per $15 \mu \mathrm{g}$ of Tf in EMEM. Five hours after transfection, $1 \mathrm{ml}$ of EMEM supplem ented with $20 \%$ fetal bovine serum (FBS) and hydrocortisone $(1 \mu \mathrm{g} / \mathrm{ml})$ was added and cultured for an additional $48 \mathrm{hr}$. The transfected cells were collected and lysed in RIPA radioimmunop recipitation assay buffer (Santa Cruz Biotechnology, Santa Cruz, CA) and Western blot analysis was performed as previously de- scribed (L. Xu et al., 1997, 1999). For the time course of p53 gene expression, $2 \times 10^{5} \mathrm{JSQ}-3$ cells were transfected with $2 \mu \mathrm{g}$ of pCMVp53 or pCMVpRo complexed with LipT. The cells were collected for Western blot analysis every $24 \mathrm{hr}$ up to 5 days after transfection and applied. To analyze the radiation effect on p53 gene expression, JSQ-3 cells were transfected with LipT-p53, or LipT-pRo ( $2 \mu \mathrm{g}$ of DNA per $2 \times 10^{5}$ cells per well in a six-well plate) for 2 days, then trypsinized and irradiated in suspension with graded doses (up to $6 \mathrm{~Gy}$ ) of ${ }^{137} \mathrm{Cs} \gamma$ rays in a J.L. Shepard and Associates Mark I irradiator (L. Xu et al., 1997). The irradiated cells were replated and cultured for 2 or 4 days before being harvested for Western blot analysis.

\section{p53 and radiation-induced apoptosis}

To study p53-induced apoptosis, JSQ-3 cells were transfected with LipT-p53 or LipT-pRo ( 1 to $3 \mu \mathrm{g}$ of DNA per $2 \times$ $10^{5}$ cells) as described above, and both the attached and floating cells were collected every day for 3 days for apoptosis staining. For radiation-induced apoptosis, the cells were transfected for 2 days, and then trypsinized and irradiated as described above. The replated cells were collected 4 days later for apoptosis staining. The collected cells were stained with the annexin V-fluorescein isothiocyanate (FITC) kit (Trevigen, Gaithersburg, MD) according to the manufacturer's protocol. The stained cells were analyzed on a flow cytometer (FACStar; Becton Dickinson, Franklin Lakes, NJ).

\section{In vivo gene transfection in nude mouse JSQ-3 xenograft model by intravenous injection of LipT-pSVb}

The transferrin-liposome, liposome-pSVb, and pSVb DNA complexes were prepared as previously described (L. Xu et al., 1997) at a ratio of $1 \mu \mathrm{g}$ of DNA per $10 \mathrm{nmol}$ of liposome per $12.5 \mu \mathrm{g}$ of transferrin. However, sterile 5\% dextrose was used in place of HBSS. The nude mouse tumor model was established by subcutaneous injection of $2.5 \times 10^{6}$ JSQ- 3 cells in the flank of 4- to 6-week-old female athymic nude mice (NCR $\mathrm{nu} / \mathrm{nu}) \cdot \mathrm{pSVb}$ DNA $(30 \mu \mathrm{g})$ complexed with Tf-liposome in a $300-\mu 1$ total volume was injected into each mouse via the tail vein, using a $1-\mathrm{m}^{1}$ syringe and a 30 -gauge needle. In the control groups, either liposome-pSVb or pSVb DNA without liposome was injected. Two and 10 days after DNA injection, the tumors as well as mouse organs were excised, cut into 1mm-thick slices, washed once with PBS, and fixed with $2 \%$ formaldehyde $-0.2 \%$ glutaraldehyde for $4 \mathrm{hr}$ at room temperature. The fixed tumor slices were washed four times with PBS, each for $1 \mathrm{hr}$, and stained with X-Gal solution plus $0.1 \%$ Nonidet $\mathrm{P}-40$ (NP-40) (pH adjusted to 8.5 ) at $37^{\circ} \mathrm{C}$ overnight. The stained tumor slices were embedded and sectioned by normal histological procedures and counterstained with nuclear fast red. Four sections per tumor were examined to evaluate the $\beta$-galactosidase gene expression, as indicated by the blue-stained cells.

\section{In vivo systemic p53 gene therapy in combination with radiation}

JSQ-3 cells $\left(2.5 \times 10^{6}\right)$ were injected subcutaneously into the lower back above the tail of athymic NCR nu/nu nude mice. Seven to 10 days later, the tumors grew to approximately $40-50$ 
$\mathrm{mm}^{3}$ at the injection site. Freshly prepared LipT-p53 or LipT-pRo containing $8 \mu \mathrm{g}$ of DNA in $300 \mu \mathrm{l}$ of $5 \%$ dextrose was intravenously injected per mouse via the tail vein twice a week for a total of five injections. Forty-eight hours after the initial intravenous injection, the animals were secured in a lead restraint that permitted only the tumor area to be exposed to $\gamma$ irradiation, and the first fractionated dose of $2.5 \mathrm{~Gy}$ of ${ }^{137} \mathrm{Cs}$ ionizing radiation was administered. Thereafter, the animals were given $2.5 \mathrm{~Gy}$ every $48 \mathrm{hr}$ to a total dose of $25 \mathrm{~Gy}$. For comparison, a group of untransfected mice, as well as a group of mice injected with LipT-p53, received no radiation. The tumor sizes were measured weekly in a blinded manner by a third party at the Georgetown University Animal Facility. All animal experiments were performed in accordance with Georgetown University institutional guidelines for the care and use of animals.

\section{Histological analysis of JSQ-3 xenografts after combination treatment}

Subcutaneous JSQ-3 tumors were excised from the animals at various times after the end of the treatment. The tumor tissues were formalin fixed, sectioned, and stained with H\&E according to common histological procedure.

\section{Statistical analysis}

The Student $t$ test and two-w ay ANOVA was performed with GraphPad Prism version 3.00 for Windows (GraphPad Software, San Diego, CA; www.graphpad.com).

\section{RESULTS}

\section{Optimization of transferrin-liposomes for transfection of head and neck cancer cells}

LipT, the liposome complex based on cationic lipids DOTAP and DOPE with transferrin, was prepared as previously described (L. Xu et al., 1997). To optimize the transfection efficiency, a plasmid designated as pCMVLuc that contains the firefly luciferase gene driven by the cytomegalovirus (CMV) promoter was employed as reporter gene. JSQ-3 cells were transfected with different DNA-lipid-Tf ratios of LipTpCMVLuc (LipT-Luc). Figure 1 shows that transferrin significantly enhanced the transfection efficiency of cationic liposomes. Under optimal conditions, i.e., a DNA:lipid:Tf ratio of $1 \mu \mathrm{g}: 10 \mathrm{nmol}: 12.5 \mu \mathrm{g}$, luciferase was expressed at $12.5 \pm 1.1$ $\mu \mathrm{g} / \mathrm{mg}$ total protein, representing $1.25 \%$ total protein, 7 - to 10 -

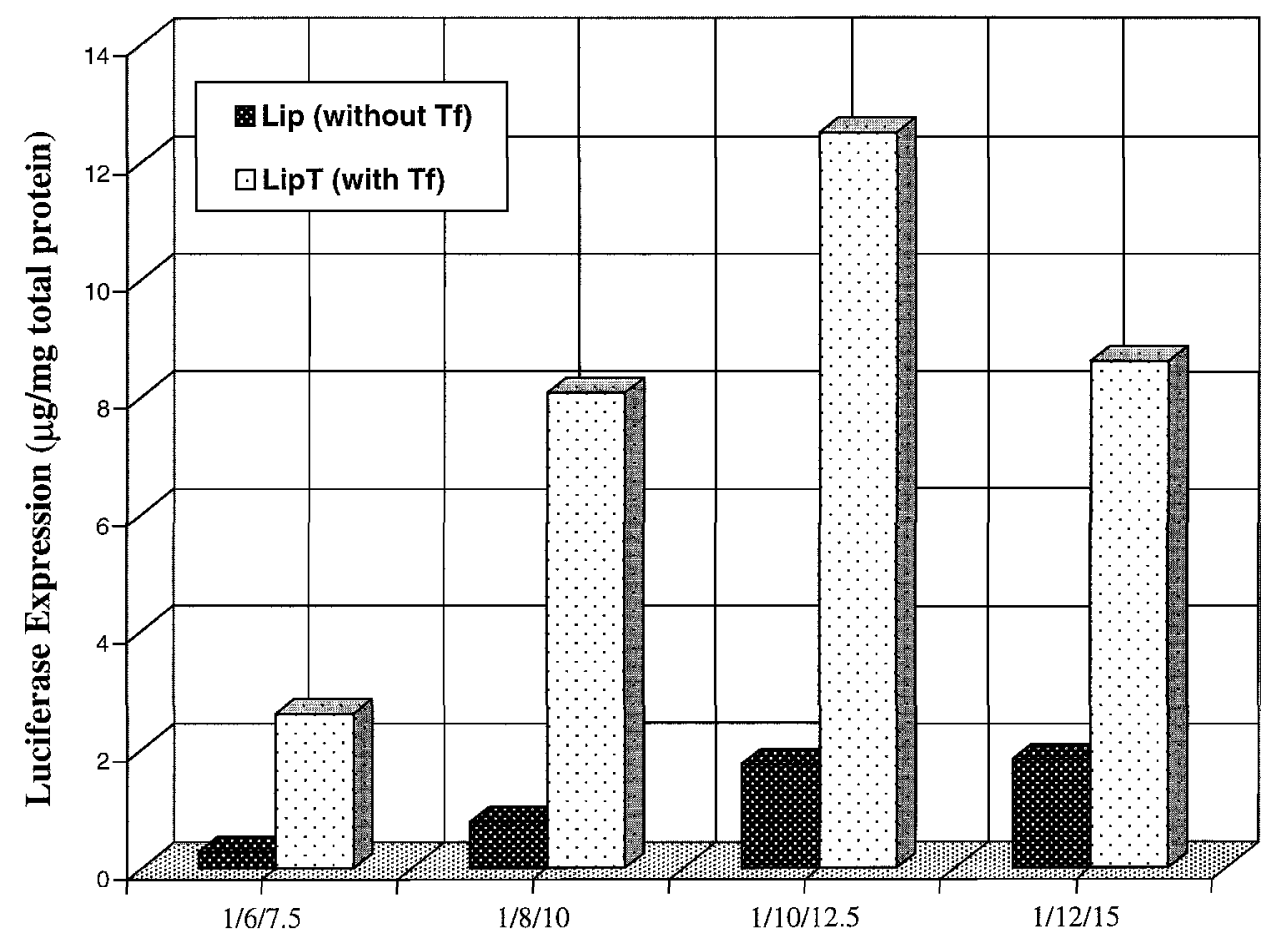

\section{Ratio of DNA/lipid/Tf $(\mu \mathrm{g} / \mathrm{nmol} / \mu \mathrm{g})$}

FIG. 1. Optimization of transferrin-liposome system by luciferase assay. In a representative experiment, $5 \times 10^{4} \mathrm{JSQ}-3$ cells per well in a 24-well plate were transfected with Tf-liposome-pCMVLuc (LipT-Luc), containing $1.0 \mu \mathrm{g}$ of plasmid DNA complexed with various amounts of cationic liposome and transferrin. Twenty-four hours later, the cells were lysed and the expressed luciferase activities were measured with the luciferase assay system, with a recombinant firefly luciferase to convert the luminometer readings of relative light units (RLU) to the amount of luciferase equivalent expressed. The results were expressed as micrograms of luciferase equivalent per milligram of total protein. The DNA:lipid:Tf ratio of $1 \mu \mathrm{g}: 10 \mathrm{nmol}: 12.5 \mu \mathrm{g}$ gave the best transgene expression: $12.5 \pm 1.1 \mu \mathrm{g} / \mathrm{mg}$ total protein, or $1.25 \%$ total protein. 
TABle 1. IN VItRo Transfection EFFICIENCIES FOR JSQ-3 CELls ${ }^{\mathrm{a}}$

\begin{tabular}{lcc}
\hline & \multicolumn{2}{c}{ Transfection efficiency $(\%)$} \\
\cline { 2 - 3 } Transfected with: & Without serum & With serum \\
\hline pSVb alone & 0 & 0 \\
Lip-pSVb & 20 & $<10$ \\
LipT-pSVb & $70-80$ & $40-50$ \\
\hline
\end{tabular}

a JSQ-3 cells (60\% confluent), cultured in a 24 -well plate, were transfected for $5 \mathrm{hr}$ with $0.5 \mathrm{ml}$ of transfection solution containing $1.2 \mu \mathrm{g}$ of $\mathrm{pSVb}$. After an additional 2 days in culture, the cells were fixed and stained with X-Gal. Transfection efficiency was calculated as the percentage of blue-stained cells. LipT-pSVb, transferrin-liposome-pSVb complex; Lip-pSVb, liposome-pSVb without transferrin. fold more than liposome alone without transferrin. The results agreed with our previous report (L. Xu et al., 1997). This ratio was used as a basic ratio for in vivo optimization below.

In the quantitative $\beta$-galactosidase assay, the JSQ-3 cells transfected under the optimal conditions, with $0.5 \mu \mathrm{g}$ of DNA per $10^{5}$ cells of LipT-pSVb, expressed $15.04 \pm 0.60 \mathrm{mU} / \mathrm{mg}$ total protein of $\beta$-galactosidase without serum, and $10.95 \pm$ $0.15 \mathrm{mU} / \mathrm{mg}$ in the presence of serum. In histochemical studies, transfection with LipT-pSVb resulted in $70-80 \%$ of the cells being transfected (see Table 1). The presence of serum during transfection slightly reduced transfection efficiency, but even with serum, $40-50 \%$ of the cells stained blue, while cationic liposome without ligand gave only $10-20 \%$ transfection efficiency. These results demonstrated that using $\mathrm{Tf}$ as a ligand significantly increased the transfection efficiency of cationic liposomes, even in the presence of serum.

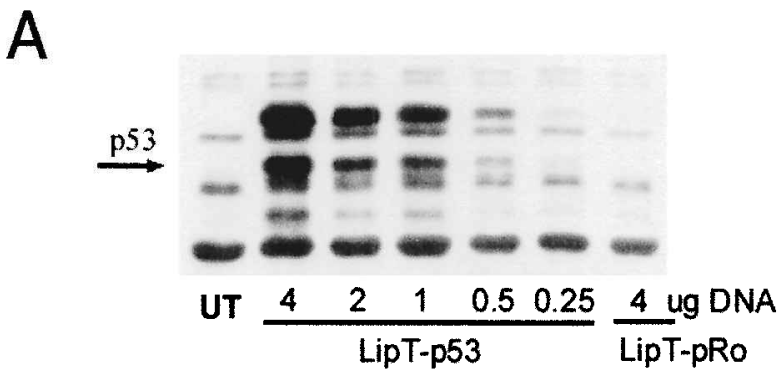

B
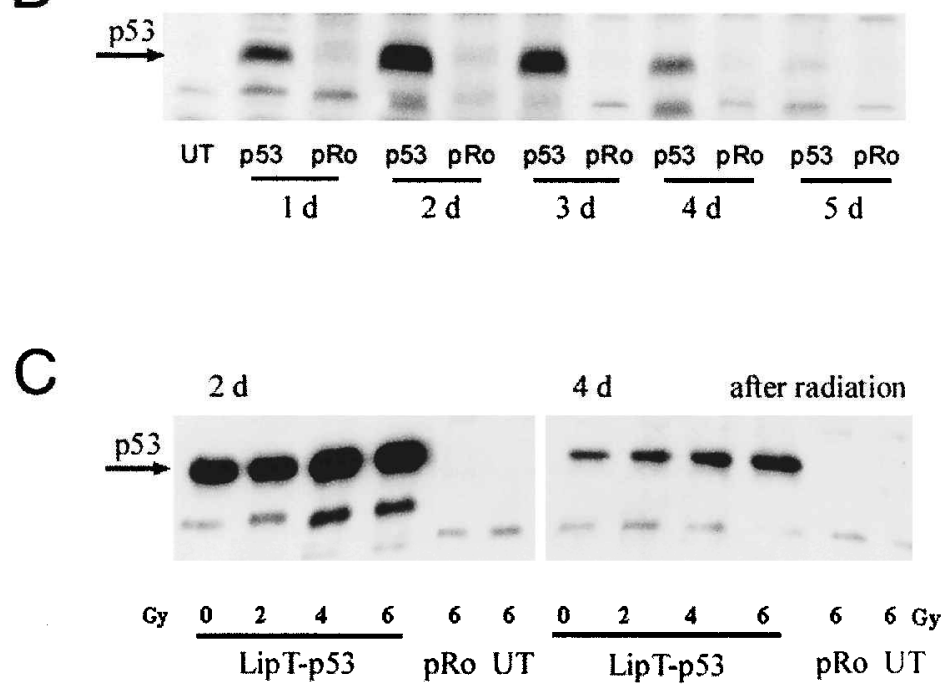

FIG. 2. Expression of exogenous wt p53 protein in LipT-p53-transfected JSQ-3 cells in vitro and its relationship to radiation. UT, Untransfected JSQ-3 cells; Lip T-p53, cells transfected with transferrin-liposome complexed to p53 plasmid pCMVp53; LipT-pRo, transferrin-liposome complexed to pCMV-pRo, the plasmid containing p53 gene in the reverse orientation. The arrows indicate exogenous wt p53. (A) DNA dose response of LipT-p53 transfection. JSQ-3 cells were transfected in a six-well plate for $5 \mathrm{hr}$ with increasing amounts of LipT-p53 or LipT-pRo as described in Materials and Methods. Forty-eight hours later the cells were collected and lysed for Western blot. A total of $40 \mu \mathrm{g}$ of protein was loaded per lane. (B) Time course of exogenous wt p53 expression in JSQ-3 cells. The cells were transfected with LipT-p53 or LipT-pRo at $2 \mu \mathrm{g}$ of plasmic DNA per $2 \times$ $10^{5}$ cells. At the indicated time, the cells were collected and lysed for Western blot analysis as described above. (C) Effect of $\gamma$ irradiation on wt p53 gene expression JSQ- 3 cells were transfected with LipT-p53 or LipT-pRo $\left(2 \mu \mathrm{g}\right.$ of DNA per $2 \times 10^{5}$ cells $)$ for 2 days, then trypsinized and irradiated at graded doses up to $6 \mathrm{~Gy}$ of ${ }^{137} \mathrm{Cs} \gamma$ rays. The irradiated cells were replated and cultured for a further 2 and 4 days before collecting for Western blot analysis. The $\gamma$ irradiation can enhance and/or stabilize the exogenous wt p53 expression in a radiation dose-dependent manner. 
Expression of exogenous wild-type p53 protein in LipT-p53-transfected JSQ-3 cells

Western blot analysis was employed to demonstrate that the LipT-transduced wt p53 was being expressed in JSQ-3 cells. As shown in Fig. 2A, transfection with increasing doses of p53 expression plasmid pCMVp53 complexed with LipT (LipT-p53) resulted in a DNA dose-dependent expression of wt $\mathrm{p} 53$, while no exogenous $\mathrm{p} 53$ expression was evident in JSQ3 cells transfected with LipT-pRo, which carries the wt p53 cDNA in reverse orientation. Wild-type p53 was expressed 24 hr after LipT-p53 transfection and peaked on the second day (Fig. 2B). Only traces of exogenous p53 were detected 5 days after transfection, indicating that LipT-mediated wt p53 expression was transient (Fig. 2B). When the cells were irradiated $48 \mathrm{hr}$ after LipT-p53 transfection, i.e., at the peak of exogenous wt p53 expression, the level of wt p53 protein increased with increasing doses of $\gamma$ irradiation (Fig. 2C). The level of p53 protein still present even 4 days postirradiation (i.e., 6 days posttransfection) indicates that $\gamma$ irradiation can enhance and/or stabilize the exogenous wt $\mathrm{p} 53$ expression in a radiation dose-dependent manner, analogous to the situation observed in cells with naturally occurring wt $\mathrm{p} 53$.

\section{Apoptosis induced by p53 transfection and $\gamma$ irradiation}

To examine the effect of wt $\mathrm{p} 53$ restoration on the induction of apoptosis, JSQ-3 cells were transfected with LipT-p53 or LipT-pRo, then stained on days 1 to 3 with annexin V-FITC, which binds specifically to phosphatidylserine present on apoptotic cells. As shown in Fig. 3, a clear induction of apoptosis is observed with LipT-mediated wt p53 restoration, in a DNA dose-dependent manner. The percentage of apoptotic cells peaked on the second day of transfection, correlating with the levels of wt $\mathrm{p} 53$ expression in the cells as revealed by Western blot (Fig. 2B). To examine the effect of irradiation on the induction of apoptosis, the transfected cells were irradiated with various doses $48 \mathrm{hr}$ after transfection. Two to 4 days later, the cells were stained with annexin V-FITC and analyzed by flow cytometry. As shown in Fig. 4A, $\gamma$ irradiation induced a significant increase in apoptosis only in LipT-p53-transfected cells, from $18.7 \%$ (0 Gy) to $38.7 \%$ (4 Gy) and $46.4 \%$ (6 Gy) 2 days after irradiation (or 4 days after transfection). No increase was observed with untransfected (UT) cells or those transfected with LipT or LipT-pRo. This increase was also radiation dose dependent and correlated, as indicated in Fig. 4B, with the wt

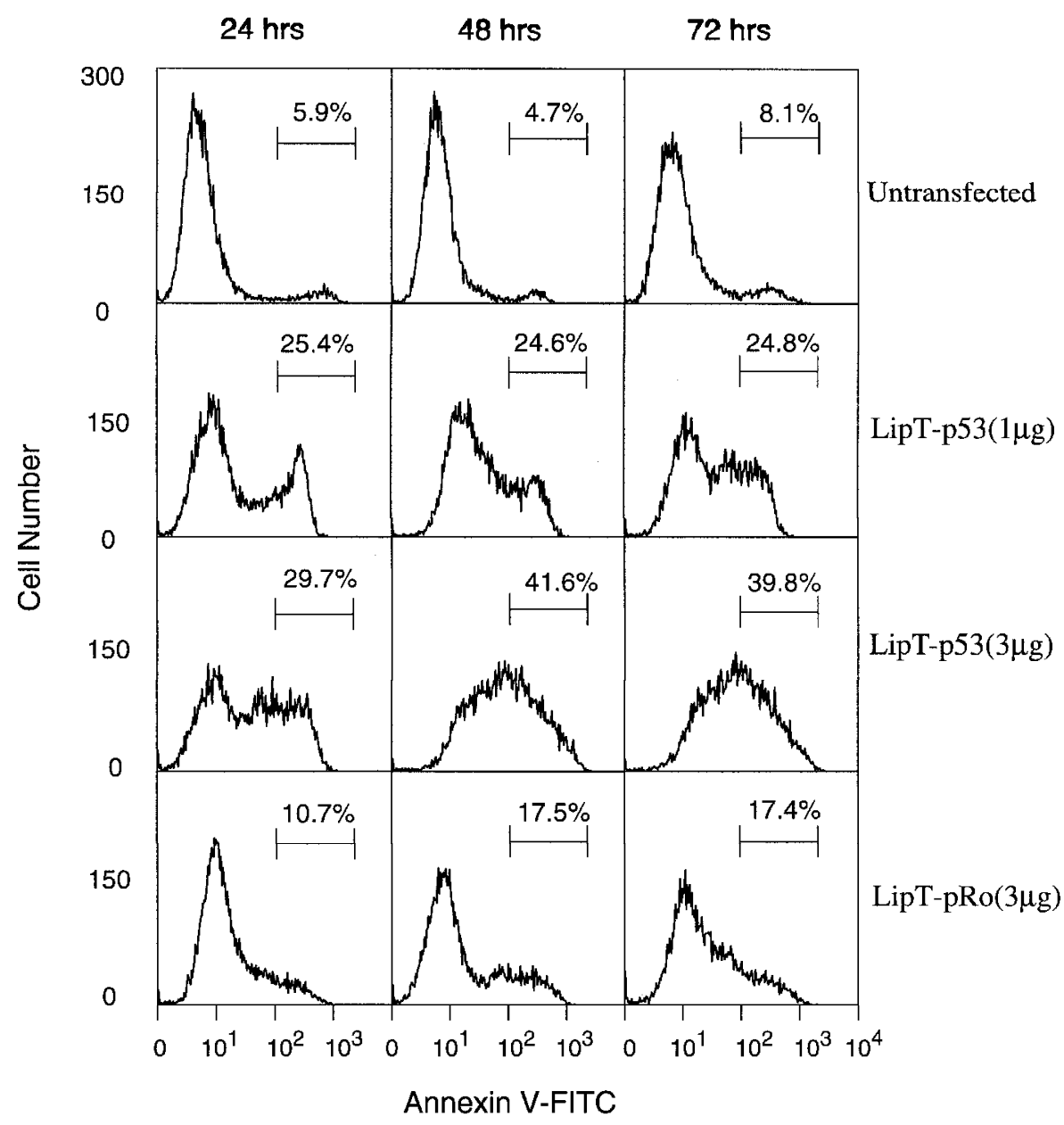

FIG. 3. LipT-p53 transfection-induce d apoptosis. JSQ-3 cells were transfected with LipT-p53 or LipT-pRo, then stained from day 1 to day 3 with annexin V-FITC, which bound specifically to phosphatidylserin e present on apoptotic cells. A clear induction of apoptosis is observed in LipT-mediated wt p53 restoration, in a DNA dose-dependent manner. 

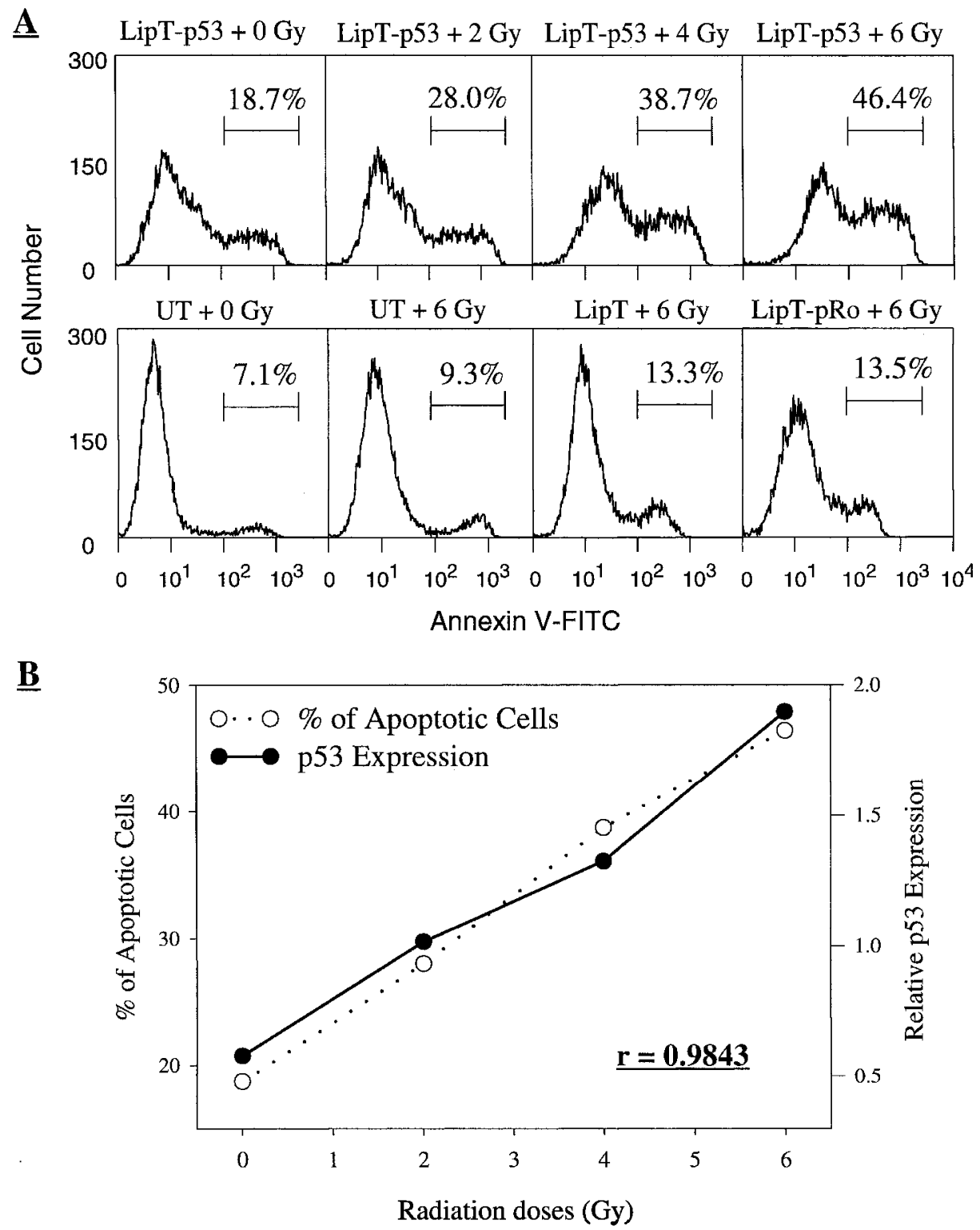

FIG. 4. The effect of irradiation on the induction of apoptosis. (A) The transfected cells were irradiated at various doses 48 hr after transfection. Two to 4 days later, the cells were stained with annexin V-FITC and analyzed by flow cytometry. $\gamma$ Irradiation induced a significant increase in apoptosis only of LipT-p53-transfected cells. (B) The increase was radiation dose dependent and correlated with the wt p53 expression levels from Western blot data.

p53 expression levels shown in Fig. 2C ( $r=0.9843)$, demonstrating that the radiation enhancement of apoptosis was proportional to the wt p53 level in cells, i.e., the more wt p53 expressed, the more apoptotic cells induced.

In vivo transfection of systemically delivered LipT-pSVb complex

To examine the ability of the Tf-containing liposomes to selectively target tumor tissue in vivo, LipT $-\mathrm{pSVb}$ or the $\mathrm{pSVb}$ plasmid alone was injected intravenously into nude mice bearing JSQ-3 subcutaneous xenografts. After either 2 or 10 days, the tumors and major organs were examined by histochemical staining with X-Gal. At 2 days, the tumors in mice injected with LipT-pSVb showed reporter gene expression representing an in vivo transfection efficiency of approximately 20-30\% with one intravenous injection (Color Plate 1A and B). In contrast, with pSVb plasmid alone, without liposome, less than $1 \%$ of the tumor cells stained for reporter gene expression (Color Plate 1D). Ten days after intravenous administration of LipT-pSVb, both the percentage of positive cells and the intensity of blue staining in the tumors were significantly reduced (Color Plate 1C), indicating that the LipT-mediated systemic transfection was transient. Vital organs in mice injected with LipT-pSVb showed staining only of macrophages, such as histocytes of the lung (Color Plate 1E) or Kupffer cells of the liver (Color Plate $1 \mathrm{~F})$, whereas the lung alveolar cells and hepatocytes remained unstained. No staining was evident in the lymphoblastic growth centers in the spleen (data not shown). In summary, the histochemical staining indicates that delivery of the reporter gene by 
A
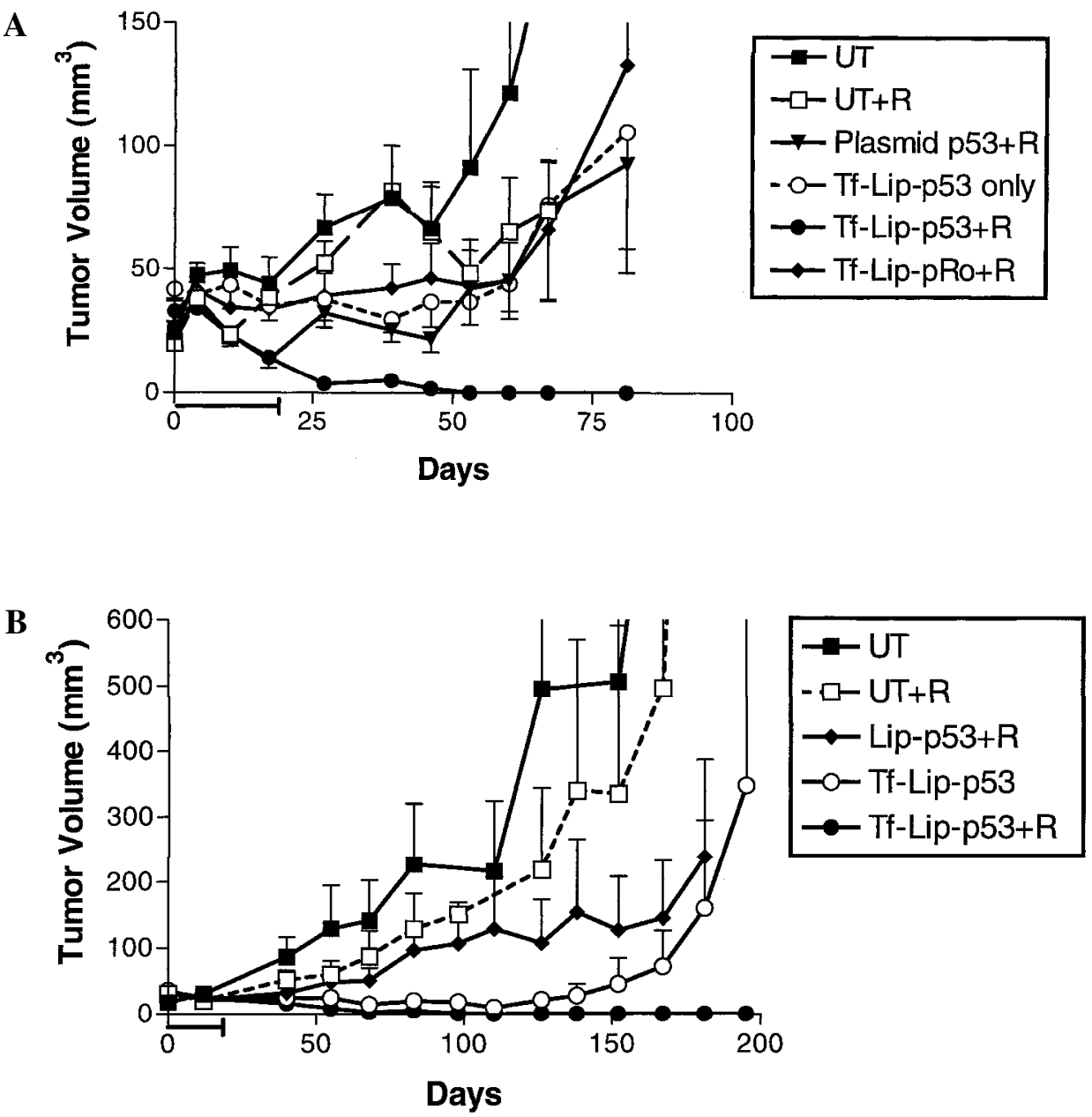

FIG. 5. Effect of the combination of LipT-p53 and radiation treatment on JSQ-3 xenografts in vivo. Day 0 represents the tumor volume prior to initiation of treatment. The last point represents 81 days (A) or 195 days (B) posttreatment. While there was no significant difference between the three control groups, the difference between the controls and the combination LipT-p53treated group was highly significant. The standard error for the combination group was negligible. The bar indicates the duration of treatment (a total of five intravenous LipT injections and $25 \mathrm{~Gy}$ of radiation).

Color Plate 1. In vivo LipT-mediated system ic transfection of $\beta$-galactosidase reporter gene in nude mouse JSQ-3 xenografts. The $\beta$-galactosidase-ex pressing cells stained blue. (A) JSQ-3 xenograft tumor from a mouse injected intravenously with LipT-pSVb, $48 \mathrm{hr}$ postinjection; (B) second, independent xenograft tumor from a mouse injected intravenously with LipT-pSVb, $48 \mathrm{hr}$ postinjection; (C) JSQ-3 tumor treated intravenously with LipT-pSVb, 10 days after injection; (D) tumor from mouse injected intravenously with pSVb alone, $48 \mathrm{hr}$ postinjection; (E) lung from a mouse carrying a JSQ-3 xenograft tumor and injected intravenously with LipT-pSVb, $48 \mathrm{hr}$ postinjection; (F) liver from a mouse carrying a JSQ-3 xenograft tumor and injected intravenously with LipT-pSVb. Bars: $50 \mu \mathrm{m}$.

Color Plate 2. Histological analysis of JSQ-3 xenografts after combination treatment Subcutaneous JSQ-3 tumors were excised from the animals at various times after and the end of treatment with radiation and/or intravenously administered LipT-p53 or LipT-pRo. The mass was formalin fixed, sectioned, and stained with H\&E. Shown is tumor or residual mass from animals that received (A) LipT-p53 plus 25 Gy, 1 day posttreatment; (B) LipT-pRo plus 25 Gy, 1 day posttreatment; (C) no intravenous treatment but 25 Gy of radiation, 1 day posttreatment; (D) LipT-p53 plus 25 Gy, 1 month posttreatment; (E and G) LipT-p53 plus 25 Gy (two individual tumors), 6 months posttreatment; (F) enlargement of the area indicated in (E), showing dead tumor cells being engulfed by Langerhans cells; $(\mathbf{H})$ LipT-p53, without radiation treatment, 6 months posttreatment; (I) LipT-pRo plus $25 \mathrm{~Gy}, 6$ months posttreatm ent. m, Normal muscle. Bars: $50 \mu \mathrm{m}$. 

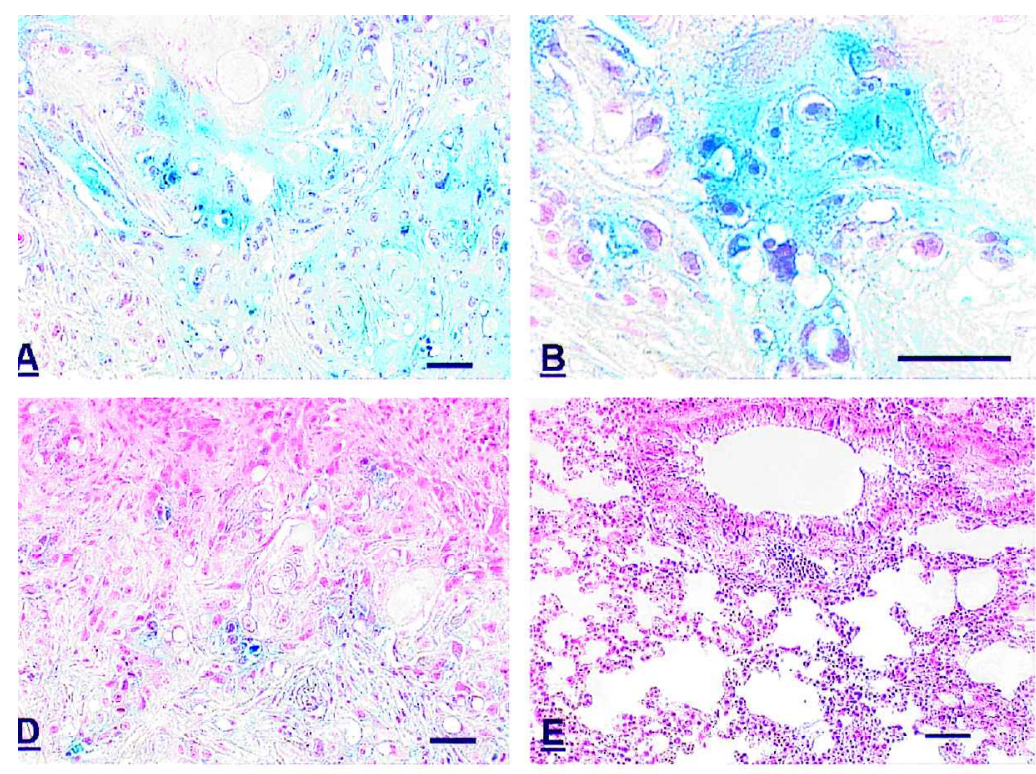

B

$4(2$

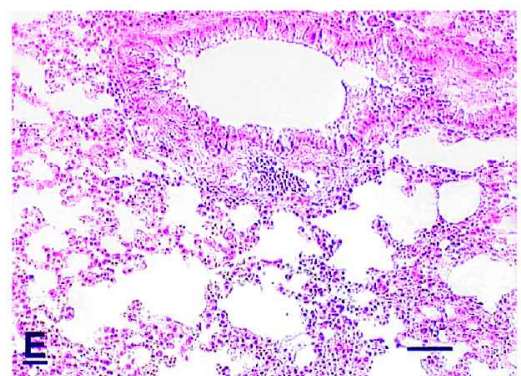

\section{Color Plate 1}

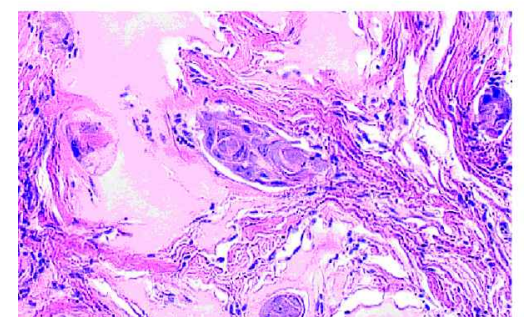

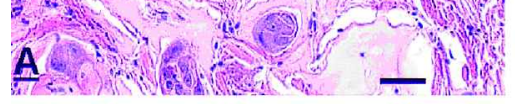
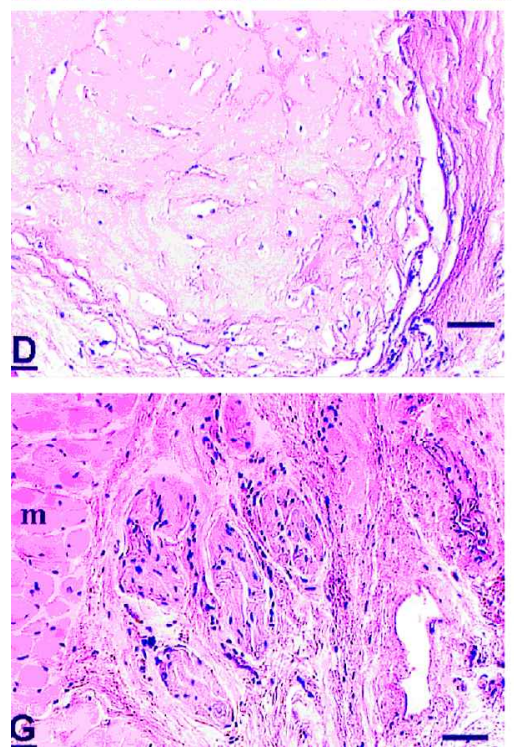
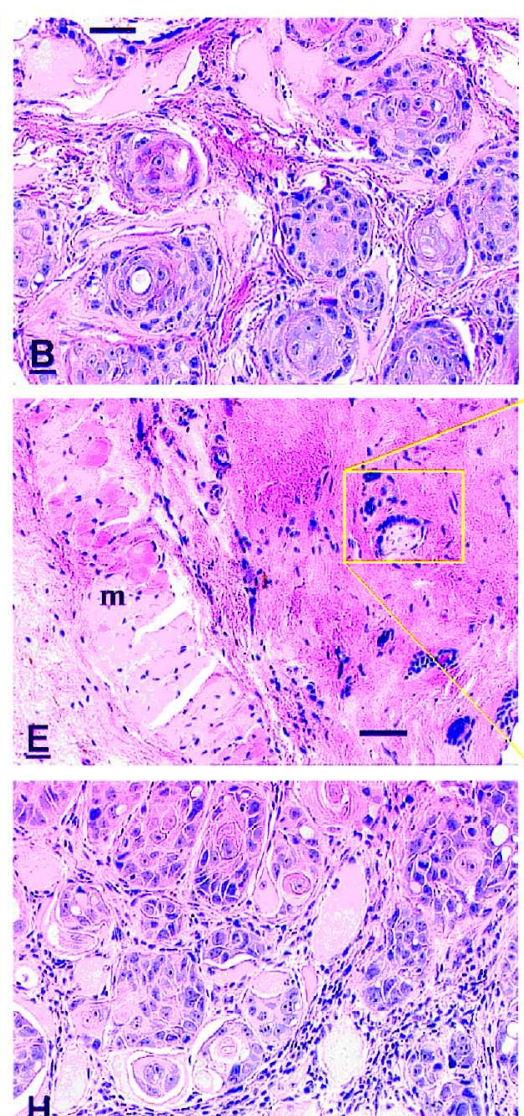

H. $3.5,402$
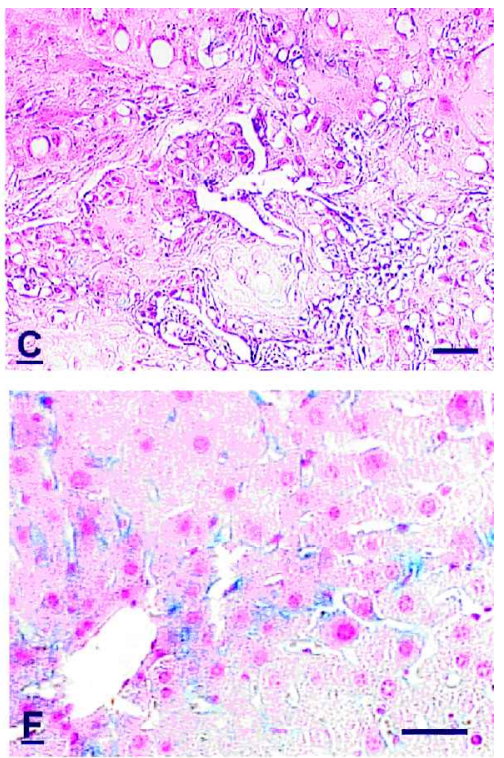

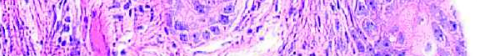

C $, 2-2=1.5:$

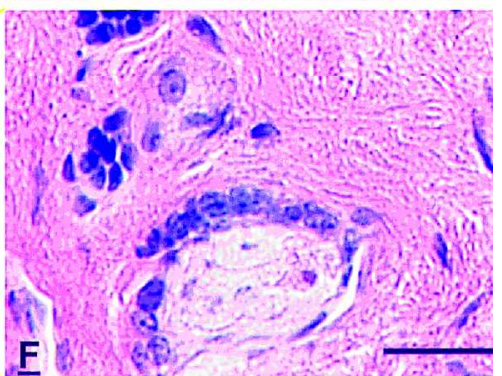

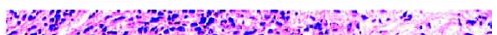
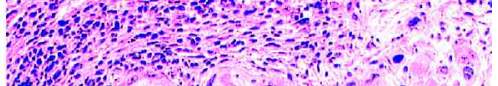

列
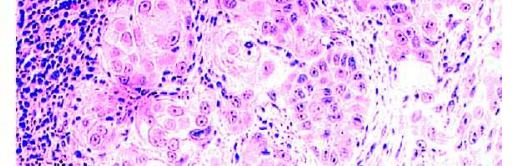

I $\quad 42^{\circ}-0^{\circ}$ 
LipT is selective, with the human xenograft being most heavily stained.

\section{Effect of the combination of LipT-p53 and radiation treatment on JSQ-3 xenografts in vivo}

Two independent experiments with SCCHN (JSQ-3) xenograft tumors have been performed with similar results (Fig. 5A and B). Mice bearing subcutaneou s JSQ-3 tumors of approxim ately $25-40 \mathrm{~mm}^{3}$ were injected, via the tail vein, with LipT-p53 twice weekly (a total of five injections) and only the tumor areas were exposed to fractionated doses $(2.5 \mathrm{~Gy})$ of $\gamma$ radiation, every other day to a total of $25 \mathrm{~Gy}$. As shown in Fig. 5A and B, short-term radiation effects on tumor growth were evident in cells transfected using the control LipT-pRo. There was only minimal tumor growth inhibition in the animals that received LipT-p53 without radiation. In contrast, in both experiments all the tumors that received the combination of LipT-p53 plus radiation exhibited virtually complete regression and showed no signs of recurrence $(p<0.001)$. In the case of the longer maintained experiment (Fig. 5B), the tumor-bearing animals in control groups had died or were humanely euthanized because of excessive tumor burden by 6 months postirradiation. However, the combination treatment group (p53 plus irrdiation) still showed no sign of tumor regrowth (Fig. 5B).

Histology of the small residual "tumor nodule" present in some of the animals, which was static at less than $10 \%$ of the original tumor volume over the entire course of the experiment, is shown in Color Plate 2. In the mice that had received LipT-p53 plus radiation, it is evident that mostly dead and dying tumor cells, many being engulfed by macrophages, and few live tumor cells remain 1 day after completion of treatment (Color Plate 2A). In contrast, although radiation effects are evident, live nests of tumor cells are clearly visible in the mice that received LipT-pRo plus radiation (Color Plate 2B). For comparison, an untreated tumor receiving radiation alone is shown in Color Plate 2C. Color Plate 2D shows, 1 month posttreatment, residual tissue at the site of the original tumor in an animal that received LipT-p53 plus radiation. No live tumor cells were evident; only scar tissue and a few multinuclear Langerhans cells were present. By 6 months posttreatment, only mature scar tissue and numerous macrophages or Langerhans cells in the process of surrounding and engulfing the remains of the dead tumor cells, are evident in the residual tumor mass from the animal in the combination treatment group (Color Plate 2E and G). An enlargem ent of this is shown in Color Plate 2F. In contrast, large areas of live cancer cells can be seen in the tumor from an animal receiving LipT-p53 without radiation (Color Plate $2 \mathrm{H}$ ) or LipT-pRo plus radiation (Color Plate 2I). These observation s were confirmed by an experienced pathologist and are consistent with our previous results in studies with intratumorally injected, adenoviral vector carrying wt p53 in conjunction with radiation (Pirollo et al., 1997). These experiments using a mouse cancer model demonstrate that the combination of transferrin-targeted systemic liposomal p53 gene therapy and conventional radiotherapy was markedly more effective than either treatment alone.

\section{DISCUSSION}

The development of viral and nonviral methods for the introduction of genes into cells in vivo has made therapeutic interventions involving gene therapy a reality. The introduction of wt p53 has been reported to suppress the growth of various types of malignancies, both in vitro and in mouse xenograft models. However, most of the in vivo results are obtained with viral p53 gene vectors and local administration (Fujiwara et al., 1994; Clayman et al., 1995; Liu et al., 1995; reviewed by Nielson and Maneval, 1998). To overcome the limitations of the viral vectors, including the immunogenicity and biosafety issues, and to realize the ultimate goal of systemic cancer gene therapy, nonviral gene delivery, especially ligand-directed tumortargeted systemic gene delivery, is an attractive alternative.

We have previously reported that wt p53 replacement by a nonviral gene delivery system, transferrin-liposome-DNA complex, sensitized SCCHN cells to radiation in vitro (L. Xu et al., 1997). This system was further optimized for systemic gene delivery in vivo. The results described above demonstrate that the transferrin-cationic liposome system can deliver genes systemically to xenograft tumors in vivo, after intravenous administration. Twenty to $30 \%$ in vivo transfection efficiency was achieved via intravenous injection of the LipT-DNA complex. The LipT-mediated p53 gene transfer apparently restored a functional apoptotic pathway in the SCCHN cells, thereby sensitizing the tumors to radiation therapy. Total tumor regression was observed in a nude mouse xenograft model of human SCCHN by a systemically delivered nonviral p53 vector in combination with conventional radiation therapy. The combination of systemic LipT-p53 gene therapy and radiotherapy for SCCHN was markedly more effective than either treatment alone. This observation is consistent with our report of radiosensitization by folate-liposome-mediated p53 gene therapy (Xu et al., 1999). Although systemic LipT-p53 alone showed suppressive effects on tum or grow th, as observed here and also by others (Lesoon-Woo et al., 1995; M. Xu et al., 1997), the tumor would ultimately recur after the treatment ceased. However, when LipT-p53 was combined with conventional radiotherapy, the established tumors regressed for up to 6 months after the end of treatment without recurrence. In the small residual "tumor nodules" that were static at less than $10 \%$ of the original tumor volume, only scar tissue and numerous macrophages or multinuclear Langerhans cells, in the process of surrounding and engulfing the remains of the dead tumor cells, were observed. Therefore, host macrophages might be involved in the treatment response (discussed below).

Lack of functional p53 results in the inability of the tumor cell to initiate programmed cell death (apoptosis) in response to DNA damage induced by radiation or other agents. Replacement of wild-type p53 should therefore restore the apoptotic response to irradiation (Liu et al., 1995). We have demonstrated previously that adenovirus mediated restoration of $\mathrm{wt}$ p53, in combination with radiation treatment, was able not only to inhibit tumor growth, but also to completely eliminate established radiation-resistant SCCHN xenograft tumors (Pirollo et al., 1997). This adenoviral wt $\mathrm{p} 53$ replacement also restored the $\mathrm{G}_{1}$ checkpoint and the $\gamma$ radiation-induced apoptotic pathway in the tumor cells, and therefore sensitized the tumors to 
radiotherapy (Chang et al., 1997). The results presented here demonstrate that (1) transferrin-liposome-mediated p53 gene delivery can efficiently restore wt p53 function and the apoptotic pathway; (2) $\gamma$ irradiation can enhance and/or stabilize exogenous wt p53 expression in a radiation dose-dependent manner; (3) radiation induces a significant increase in p53-dependent apoptosis; and (4) this increase is radiation dose dependent and correlates with the exogenous wt p53 level in tumor cells. Further work is now underway to analyze the LipT-p53-mediated changes in p53 downstream effectors or related molecules, e.g., p21 WAF1, Bcl-2, MDM2, p19ARF, etc., as well as cell cycle-related analysis.

The host defense system plays as important role in interactions between therapeutic regimens and target tissue, especially in the case of cancer gene therapy. With transferrin-targeted cationic liposome gene delivery, although only a fraction of tumor cells were transfected, the combination therapy resulted in tumor regression without recurrence 6 months after the treatment stopped. Antiangiogenesi s may be an important mechanism involved in the system, as observed by M. Xu et al. (1997). Liu et al. (1999) reported that systemic p53 gene delivery by cationic liposome inhibited angiogenesis by inducing endogenous thrombospondin-1 expression. Natural killer (NK) cells, which are abundant in the nude mouse, may also play a role in the tumor regression, as suggested by Hall et al. (1998). As mentioned above, host macrophages are involved in the tumor response. Systemic LipT-p53 gene therapy plus radiation resulted in tumor cell death, likely through the induction of apoptosis. These apoptotic cells were phagocytosed by macrophages and possibly also by dendritic cells. The macrophages and dendritic cells can then serve as antigen-presenting cells to stimulate the host immune system (Lotze and Jaffe, 1999). In the case of the athymic nude mouse, which lacks $\mathrm{T}$ lymphocytes, NK cells may be involved. Peron et al. (1999) suggested a dendritic cell-NK cell link, in antitum or therapy. Currently, we are testing LipT-p53 systemic gene therapy in immune-competent syngenic tum or models to explore the immunological and molecular mechanisms.

In conclusion, the transferrin-liposome system is a highly efficient and tumor-specific gene delivery system both in vitro and in vivo. The replacem ent of wild-type p53 by LipT-p53 restored the wt p53 functional apoptotic pathway. The systemic delivery of LipT-p53 to established human SCCHN xenografts markedly sensitized the tumors to radiation therapy. The combination of systemic p53 gene therapy and conventional radiotherapy resulted in tumor regression and inhibition of their recurrence long-term. This strategy may provide a more effective treatment modality for human head and neck cancer.

\section{ACKNOWLEDGMENTS}

We thank Ms. Tina Wilson for assistance with the in vivo animal studies, Dr. Laiman Xiang for assistance with tissue culture, Drs. Karen Creswell and Wen-Hui Zhu for assistance with flow cytometery analysis, and Dr. Williams Alexander for his critical review of the manuscript. This work was supported in part by NCI Grant R01 CA 45158 (E.C.) and Synergene Therapeutics, Inc.

\section{REFERENCES}

BATES, S., and VOUSDEN, K.H. (1999). Mechanisms of p53-mediated apoptosis. Cell. Mol. Life Sci. 55, 28-37.

BRISTOW, R.G., BENCHIMOL, S., and HILL, R.P. (1996). The p53 gene as a modifier of intrinsic radiosensitivity: implications for radiotherapy. Radiother. Oncol. 40, 197-223.

CHACKAL-ROY, M., NIEMEYER, C., MOORE, M., and ZETTER, B.R. (1989). Stimulation of human prostatic carcinoma cell growth by factors present in human bone marrow. J. Clin. Invest. 84, 43-50. CHANG, E.H., JANG, Y.J., HAO, Z., MURPHY, G., RAIT, A., FEE, W.E., Jr., SUSSMAN, H.H., RYAN, P., CHIANG, Y., and PIROLLO, K.F. (1997). Restoration of the G1 checkpoint and the apoptotic pathway mediated by wild-type p53 sensitizes squamous cell carcinoma of the head and neck to radiotherapy. Arch. Otolaryngol. Head Neck Surg. 123, 507-512.

CHENG, P.W. (1996). Receptor ligand-facilitated gene transfer: Enhancement of liposome-mediated gene transfer and expression by transferrin. Hum. Gene Ther. 7, 275-282.

CHRISTIANO, R.J., and CURIEL, D.T. (1996). Strategies to accomplish gene therapy via the receptor-mediated endocytosis pathways. Cancer Gene Ther. 3, 457-497.

CLAYMAN, G.L., EL-NAGGAR, A.K., ROTH, J.A., ZHANG, W.W., GOEPFERT, H., TAYLOR, D.L., and LIU, T.J. (1995). In vivo molecular therapy with p53 adenovirus for microscopic residual head and neck squamous carcinoma. Cancer Res. 55, 1-6.

DIMERY, I.W., and HONG, W.K. (1993). Overview of combined modality therapies for head and neck cancer. J. Natl. Cancer Inst. 85, 95-111.

ELLIOTT, R.L., ELLIOTT, M.C., WANG, F., and HEAD, J.F. (1993). Breast carcinoma and the role of iron metabolism. A cytochemical, tissue culture, and ultrastructural study. Ann. N.Y. Acad. Sci. 698, 159-166.

FELGNER, P.L., TSAI, Y.J., SUKHU, L., WHEELER, C.J., MANTHORPE, M., and MARSHALL, J. (1995). Improved cationic lipid formulations for in vivo gene therapy. Ann. N.Y. Acad. Sci. 772, 126-139.

FUJIWARA, T., GRIMM, E.A., MUKHOPADHYAY, T., ZHANG, W.W., OWEN-SCHAUB, L.B., and ROTH, J.A. (1994). Induction of chemosensitivity in human lung cancer cells in vivo by adenovirusmediated transfer of the wild-type p53 gene. Cancer Res. 54, 2287-2291.

GRAYHACK, J.T., WENDEL, E.F., OLIVER, L., and LEE, C. (1979). Analysis of specific proteins in prostatic fluid for detecting prostatic malignancy. J. Urol. 121, 295-299.

HALL, S.J., SANFORD, M.A., ATKINSON, G., and CHEN, S.H. (1998). Induction of potent antitumor natural killer cell activity by herpes simplex virus-thymidine kinase and ganciclovir therapy in an orthotopic mouse model of prostate cancer. Cancer Res. 58, 3221-3225.

HUANG, L., and VIROONCHATAPAN, E. (1990). Introduction. In Non-Viral Vectors for Gene Therapy. L. Huang, M.C. Hung, and E. Wagner, eds. (Academic Press, San Diego, CA) pp. 3-22.

INOUE, T., CAVANAUGH, P.G., STECK, P.A., BRUNNER, N., and NICOLSON, G.L. (1993). Differences in transferrin response and numbers of transferrin receptors in rat and human mammary carcinoma lines of different metastatic potentials. J. Cell Physiol. 156, 212-217.

KEER, H.N., KOZLOWSKI, J.M., and TSAI, M.C. (1990). Elevated transferrin receptor content in human prostate cancer cell lines assessed in vitro and in vivo. J. Urol. 143, 381-385.

LANDIS, S.H., MURRAY, T., BOLDEN, S., and WINGO, P.A. (1998). Cancer statistics, 1998. CA Cancer J. Clin. 48, 6-29.

LEE, J.M., and BERNSTEIN, A. (1993). p53 mutations increase resistance to ionizing radiation. Proc. Natl. Acad. Sci. U.S.A. 90, 5742-5746. 
LESOON-WOOD, L.A., HO KIM, W., KLEINMAN, H.R., WEINTRAUB, B.D., and MIXSON, A.J. (1995). Systemic gene therapy with $\mathrm{p} 53$ reduces growth and metastases of a malignant human breast cancer in nude mice. Hum. Gene Ther. 6, 395-405.

LIU, T.J., EL-NAGGAR, A.K., McDONNELL, T.J., STECK, K.D., WANG, M., and TAYLOR, D.L. (1995). Apoptosis induction mediated by wild-type p53 adenoviral gene transfer in squamous cell carcinoma of the head and neck. Cancer Res. 55, 3117-3122.

LIU, Y., THOR, A., SHTIVELMAN, E., CAO, Y., TU, G., HEATH, T.D., and DEBS, R.J. (1999). Systemic gene delivery expands the repertoire of effective antiangiogenic agents. J. Biol. Chem. 274, $13338-13344$.

LOTZE, M.T., and JAFFE, R. (1999). Dendritic cells in disease: Cancer. In Dendritic Cells: Biology and Clinical Applications. M.T. Lotze and A.W. Thomson, eds. (Academic Press, San Diego, CA) pp. 325-338.

MCILWRATH, A.J., VASEY, P.A., ROSS, G.M., and BROWN, R. (1994). Cell cycle arrests and radiosensitivity of human tumor cell lines: dependence on wild-type p53 for radiosensitivity. Cancer Res. 54, 3718-3722.

MENG, R.D., and EL-DEIRY, W.S. (1999). Tumor suppressor genes as targets for cancer gene therapy. In Gene Therapy of Cancer. E.C. Lattime and S.L. Gerson, eds. (Academic Press, San Diego, CA), pp. $3-20$.

MIYAMOTO, T., TANAKA, N., EISHI, Y., and AMAGASA, T. (1994). Transferrin receptor in oral tumors. Int. J. Oral Maxillofac. Surg. (Denmark) 23, 430-433.

NIELSON, L.L., and MANEVAL, D.C. (1998). p53 tumor suppressor gene therapy for cancer. Cancer Gene Ther. 5, 52-63.

O'CONNOR, P.M., JACJMAN, J., JONDLE, D., BHATIA, K., MAGRATH, I., and KOHN, K.W. (1993). Role of the p53 tumor suppressor gene in cell cycle arrest and radiosensitivity of Burkitt's lymphoma cell lines. Cancer Res. 53, 4776-4780.

PERON, J.M., ESCHE, C., SUBBOTIN, V.M., MALISZEWSKI, C., LOTZE, M.T., and SHURIN, M.R. (1998). FLT3-ligand administration inhibits liver metastases: Role of NK cells. J. Immunol. 161, 6164-6170.

PIROLLO, K.F., HAO, Z., RAIT, A., JANG, Y.J., FEE, W.E., Jr., RYAN, P., CHIANG, Y., and CHANG, E.H. (1997). p53 mediated sensitization of squamous cell carcinoma of the head and neck to radiotherapy. Oncogene 14, 1735-1746.

ROSSI, M.C., and ZETTER, B.R. (1992). Selective stimulation of prostatic carcinoma cell proliferation by transferrin. Proc. Natl. Acad. Sci. U.S.A. 89, 6197-6201.

ROTH, J.A. (1998). Restoration of tumour suppressor gene expression for cancer. Forum (Genova) 8, 368-376.

RULEY, H.E. (1996). p53 and response to chemotherapy and radio- therapy. In Important Advances in Oncology 1996. V.T. DeVita, S. Hellman, and S.A. Rosenberg, eds. (Lippincott-Rave n, Philadelphia, PA), pp. 37-56.

TEMPLETON, N.S., LASIC, D.D., FREDERIK, P.M., STREY, H.H., ROBERTS, D.D., and PAVLAKIS, G.N. (1997). Improved DNA:liposome complexes for increased systemic delivery and gene expression. Nature Biotechnol. 15, 647-652.

THORSTENSEN, K., and ROMSLO, I. (1993). The transferrin receptor: Its diagnostic value and its potential as therapeutic target. Scand. J. Clin. Lab. Invest. Suppl. 215, 113-120.

van DONGEN, G.A., and SNOW, G.B. (1997). Prospects for future studies in head and neck cancer. Eur. J. Surg. Oncol. 23, 486-491.

[UNREF1WAGNER, E. (1999). Ligand-polycation conjugates for receptor-targeted gene transfer. In Non-Viral Vectors for Gene Therapy. L. Huang, M.C. Hung, and E. Wagner, eds. (Academic Press, San Diego, CA) pp. 208-227.

WEICHSELBAUM, R.R., BECKETT, M.A., VOKES, E.E., HALLAHAN, D.E., HARAF, D.J., and BRACHMAN, D.G. (1993). Cellular and molecular basis for radioresistance in head and neck cancer. Head Neck Cancer III, 399-407.

XU, L., PIROLLO, K.F., and CHANG, E.H. (1997). Transferrin-liposome-mediated p53 sensitization of squamous cell carcinoma of the head and neck to radiation in vitro. Hum. Gene Ther. 8, 467-475.

XU, L., PIROLLO, K.F., RAIT, A., MURRAY, A.L., and CHANG, E.H. (1990). Systemic p53 gene therapy in combination with radiation results in human tumor regression. Tumor Targeting 4, 92-104.

XU, M., KUMAR, D., SRINIVAS, S., DETOLla, L.J., YU, S.F., STASS, S.A., and MIXSON, A.J. (1997). Parenteral gene therapy with $p 53$ inhibits human breast tumors in vivo through a bystander mechanism without evidence of toxicity. Hum. Gene Ther. 8, $177-185$.

Address reprint requests to: Dr. Esther H. Chang Lombardi Cancer Center The Research Building/E420 Georgetown University Medical Center 3970 Reservoir Road NW Washington, D.C. 20007

E-mail: change@gunet.georgetow n.edu

Received for publication July 20, 1999; accepted after revision September 20, 1999. 